\title{
Ageing and health across the world
}

\author{
Tianji Cai ${ }^{1} \cdot$ Jiehua $\mathrm{Lu}^{2} \cdot$ Qiushi Feng ${ }^{3}$ \\ Published online: 7 December 2021 \\ (c) China Population and Development Research 2021
}

The past few decades have witnessed a global trend of population aging as a result of declines in fertility and improvements in health and longevity. While more elder people can live longer, aging has brought substantial impacts on healthcare and welfare systems at societal level, as well as changes on individual physical and psychological health and family ecosystems. Meanwhile, the Global Burden of Disease, a study conducted by the World Health Organization and World Bank, shows that large amount of increase of disabled elderly is predicable in almost all regions of the world.

Although extensive research efforts have been devoted to aging studies, the importance of aging cannot be overstated before global awareness is reached and policies are made to address its challenges and opportunities. The happening COVID-19 pandemic again imposes extra pressure on the older population-not only directly on their physical health as many of them suffer from chronic diseases that can lead to rapid physical health deterioration if infected, but also indirectly on psychological well-being because they are more vulnerable to stressful situations and are more dependent to supporting networks and caregivers. Thus, public health measures adopted by governments around the world, such as social distancing and stay-at-home, might further aggravate their social isolation and loneliness that increase stress and anxiety. Therefore, for evidence-based policy implications and interventions, studies that can bring new perspective and empirical evidence to fully understand the trend of global and local aging during the current COVID-19 pandemic are needed.

To advance knowledge of aging and address the challenges brought by the pandemic, the special issue of China Population and Development Studies contains a collection of articles that cover both theoretical development and empirical analysis in studies of aging and health. These articles feature various wide ranging and

Tianji Cai

tjcai@um.edu.mo

1 University of Macau, Macau, China

2 Peking University, Beijing, China

3 National University of Singapore, Singapore, Singapore 
relevant topics such as quality of life, physical and mental health, and impact of COVID-19 on old adults.

Jasmon Hoh, Wan Ting and Qiushi Feng's paper focuses on regional disparities on bathing difficulties among older adults in China. Critical to daily functioning, Activities of Daily Living (ADL) items, including bathing, dressing, toileting, transferring, continence, feeding, have been commonly used in demographic analysis for measuring a person's functional status. Besides its connection to poor quality of life, the inability to perform those activities without assistance is considered an effective predictor for the need of alternative living arrangements, such as hospitalization, nursing home, etc., and life span. Although the prime focus of ADL is to measure physical impairment, previous studies have suggested that environmental factors could also contribute to the changes of ADL.

Ting and Feng's work extends the current understanding of how environmental factors influence the ADL dependency among Chinese older adults. Utilizing eight waves of the Chinese Longitudinal Health Longevity Survey (CLHLS) from 1998 to 2018, the authors found that substantial differences exist across geographical regions in bathing disability among older adults in Mainland China. Specifically, older adults residing in the northern regions were associated with significantly higher chance to experience bathing disability compared to their counterparts who live in the South even after controlling for confounding factors, such as sociodemographic variable, socioeconomic status, health status, and health behaviors. Within the Northern regions including Shaanxi, Beijing, Tianjin, Hebei, Shanxi, Liaoning, Jilin, and Heilongjiang provinces, elderly who live in the Northeast, such as Liaoning, Jilin, and Heilongjiang provinces, experienced highest likelihood of bathing disability. More importantly, the regional disparity only existed for bathing disability, but not the other ADL items, which suggests the potential heterogeneity of environmental influences on the ADL items. In particular, the authors argue that the large regional disparity might relate to both climatic and economic factors. Besides presenting empirical evidence that calls for caution of using ADL items as homogeneous elements, the study also provides important policy implications. For instance, as bathing is a highly environment-dependent activity, more resources are needed to improve the bathing environment for reducing the prevalence of bathing disability among older adults.

The work done by Shafayat Sultan, Mohammad Mainul Islam, and Mohammad Bellal Hossain explore the changes of health disparities among elderly during the pandemic in Bangladesh. Using content analysis, the authors analyzed 102 content collected from various online and printed articles published in newspapers, journals, and other relevant sources and found that people in Bangladesh experienced increased health risks, deteriorated mental health, and poor health system functioning during the pandemic. In particular, while the health sector of Bangladesh is struggling to provide care to critical patients, elderly are facing extra challenges during the pandemic period, such as disruption of health enhancing routine, increased health risks due to presence of multi-morbidity, lack of emergency and older people friendly health care facilities, etc.

Available evidence shows that in Bangladesh, the older population is rapidly increasing, living with various disadvantaged socio-economic conditions, including 
inadequate access and healthcare services, which make them highly vulnerable and more likely to have adverse health outcomes. Aiming to develop a framework for age-friendly hospital initiatives for health services in a low-resource setting, the authors call for greater efforts to improve the health system of Bangladesh to cope with the increasing demand of the older population.

Xiaodan Wei's paper reviews the recent studies on health for elderly, and argues that since aging process involves physiological, social, economic, and psychological changes, perspectives based on single discipline might not be adequate to provide good understanding of health issues for elderly. A holistic framework that can take into account impacts throughout life course such as social, behavioral, psychological, environmental, and genetic factors is needed for studying health issues of elderly. Health of elderly is not only an outcome at individual level, but also an influential factor that plays a role in social processes across the macro, meso and macro levels. Correspondently, the author pinpoints two main analytical strategies to utilize this holistic framework. For example, the first one is to use health as an outcome, exploring the direct effects of multilayer factors drawn from social, behavioral, psychological, environmental, and genetic perspectives; while the second one emphasizes on the dynamic nature of health, because health of elderly could influence family ecosystems such as living arrangement and economic decisions, as well as healthcare systems that cope up with epidemiological transition at societal level. The author also identifies a few existing issues that limit the study of health for elderly in China, such as lack of data sharing consortium that links data obtained from multiple sources, and training programs that help social scientist to utilize tools from other disciplines.

Nasim Ahamed Mondal's paper examines the variation of Quality Of Life (QOL) in China, India, Ghana, South Africa, Mexico, and Russia using the data obtained from Study on Global Ageing and Adult Health. QOL score has been frequently used as an indicator of wellbeing among the older population. Studies have reported that QOL varies little across countries, however, there is considerable variation across the socioeconomic and demographic characteristics within each of countries.

The chance of elder people in developing countries to access health infrastructure, geriatric system and social security varies by resources they possessed due to undeveloped public healthcare system. Thus, socio-economic characteristics of the elder population contribute to the variation of their QOL score in many developing countries. For example, the rapid expansion of the private sector into the countries like India, South Africa and Mexico, where the private sector plays a major role, is unlikely to benefit the bulk of the elders who lack health care support, which in turn does not reduce their QOL score variation; while financial support significantly contributes to QOL in terms of policy intervention in those countries. Therefore, to achieve the goal of healthy lives and wellbeing, governments in these countries need to spend resources on health care, financial and social security to improve QOL among elderly.

Taking advantage of four waves of the Chinese Longitudinal Healthy Longevity Survey (CLHLS) from 2005 to 2014, Jiehua Lu, and Keqi Liu explore the relationship between the quality of sleep and mental health among Chinese elderly. Their findings highlight the positive relationship between quality of sleep and mental health status of the elderly. Contrary to what is widely believed that quality of sleep deteriorates as 
people age, their results obtained from four waves of longitudinal studies show that the elderly in China generally have relatively good quality of sleep with little variation over years. After controlling demographic and health behavioral characteristics, respondents who reported better quality of sleep show higher level of metal health status. The authors also implement fixed effect model to control omitted variable problem, and argue that the fixed effect model combined with the longitudinal design of CLHLS can clarify potential causal relationship between quality of sleep and mental health.

In spite of the fact that the fixed effect model provide more robust inference against omitted variable compared to regular ordinary least square regression, it is still a stretch to claim that the results obtained from the fixed effect model imply causality. More robustness check and sensitivity analysis are needed to evaluate the model specification, especially the self-reported mental health and quality of sleep are subject to measurement error and social desirability biases.

\section{Declarations}

Conflict of interest The authors claim no conflict of interest.
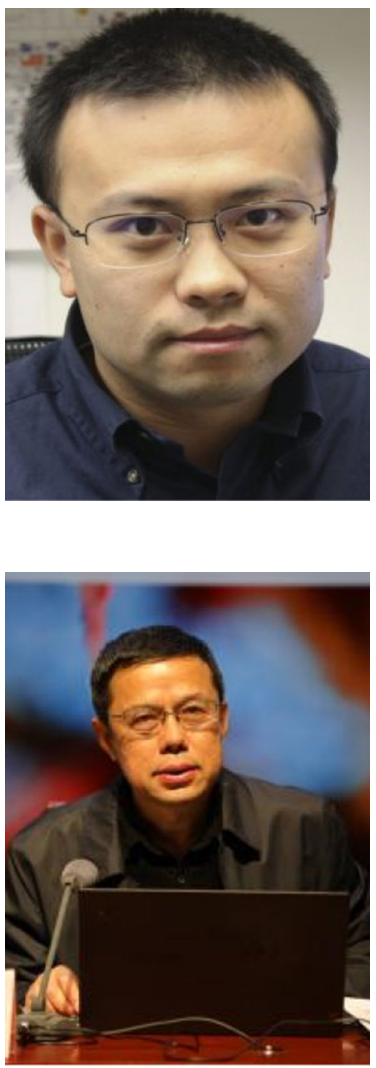

Jiehua Lu is a Professor at the Department of Sociology, Peking University and also deputy director of the Center for Healthy Aging and Development Studies, Peking University.
Tianji Cai is an Associate Professor at University of Macau. His research interests concentrate on new forms of data and new methods of analysis. \\ 包 Springer}




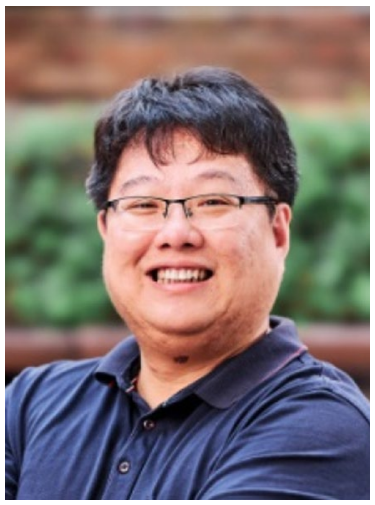

Qiushi Feng is an Associate Professor in National University of Singapore (NUS). He is Deputy Head of Department of Sociology and Deputy Director of the Centre for Family and Population Research (CFPR) in NUS. 